\title{
COMPLEX REFRACTIVE INDEX OF CONCENTRATED MAGNETIC FLUIDS
}

\author{
Constantine Yerin ${ }^{1, *}$, Victoria Lykhmanova ${ }^{1}$, and Marina Erina $^{1}$ \\ ${ }^{1}$ North Caucasian Federal University, Stavropol, Russia
}

\begin{abstract}
The spectra of the real and imaginary parts of the refractive index of magnetic fluids based on kerosene with a solids phase concentration of $1 \%$ to $35 \%$ are experimentally measured. The comparison of calculations results have been made according to Maxwell-Garnett and Bruggeman's models of effective medium approximation. The influence of refractive index spectra on the extinction of light in magnetic fluids is shown.
\end{abstract}

\section{Introduction}

Magnetic fluids are colloidal systems of magnetic nanoparticles in a liquid medium with significant magneto-optical properties. In magnetic fluids birefringence, dichroism, anisotropic light scattering, and the change in transparency are observed under the action of external magnetic field. With these spectacular and useful magneto-optical properties, magnetic fluid show promise in the development of various devices, such as optical switches, tuneable gratings, filters, magnetic-field sensors, and static or dynamic displays, etc [1]. The refractive index of magnetic nanoparticles is one of the most important parameters that determine various optical effects in magnetic fluids. The complex refractive index of magnetite for the infrared region is usually assumed to be equal to $\widetilde{n}=2.2-0.58 i$ [2]. The other indexes in visible region of the spectrum, are known to differ in larger [3] as well as in smaller [4] extend. The influence of the magnetic field makes it possible to change the refractive index of a magnetic fluid and it gives prospects for the creation of magnetically tuneable optical devices. $[5,6]$.

Magnetic emulsions based on magnetic fluid are the object of a large number of experimental and theoretical studies [7]. In such systems, a number of unusual optical effects is associated with the formation of structures from microdroplets in electric and magnetic fields, as well as with deformation of droplets are observed $[8,9]$. Data of the complex refractive index of magnetic microdrops from a concentrated magnetic fluid are necessary for creating the models of optical effects in magnetic emulsions.

In this paper, we present experimental results of complex refractive index in the visible region for kerosene based magnetic fluids with various concentrations of the magnetite. According to the experiment, the refractive index of magnetite nanoparticles is determined using known approximations of effective medium theory. The experiment was carried out in attempt to value the refractive index for magnetite nanoparticles extrapolation on the ground of common models.

\section{Experiments}

To determine the complex refractive index of magnetic fluids, we used the "Ellipse-1891" spectral ellipsometric complex, which made it possible to measure ellipsometric parameters in the wavelength range 350 $1050 \mathrm{~nm} .16$ samples of a magnetic fluid with volume concentrations in the range of $1-35 \%$ were measured. The initial sample for all samples was a magnetic fluid with a concentration of $14 \%$, from which all other samples were obtained by dilution or evaporation. The most concentrated magnetic fluids with a concentration of more than $28 \%$ were pasty-like or solid samples.

The method of ellipsometry consists in determining the refractive index of the surface from the measured ellipsometric parameters. Ellipsometry measures the complex reflectance ratio $\rho$ of a system, which may be parametrized by the amplitude component $\psi$ and the phase difference $\Delta$. The polarization state of the light incident upon the sample may be decomposed into an $s$ and a $p$ - component (the $s$ - component is oscillating perpendicular to the plane of incidence and parallel to the sample surface, and the $p$ - component is oscillating parallel to the plane of incidence, see. Fig. 1). The reflection coefficients of the $s$ - and $p$ - components of light are denoted by $R_{\mathrm{s}}$ and $R_{\mathrm{p}}$, respectively. The relationship between the basic ellipsometric parameters $(\psi, \Delta)$ and the complex reflection coefficients $\left(R_{\mathrm{s}}, R_{\mathrm{p}}\right)$ is given by the basic equation of ellipsometry [10]:

\footnotetext{
Corresponding author: exiton@inbox.ru
} 


$$
\rho=\tan \psi \exp (i \Delta)=\frac{R_{p}}{R_{s}} .
$$

In case of reflection from a semi-infinite medium, equation (1) can be solved in an analytical form and the refractive index of the reflecting surface:

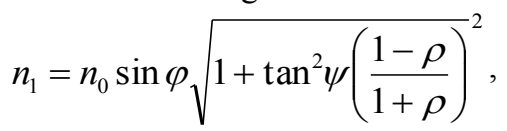

where $\varphi$ is the angle of light incidence, $n_{0}$ is the refractive index of the second medium (air in our case).

Fig. 2 shows the spectral dependences of the real part of the complex refractive index for three samples of magnetic fluids of various concentrations. Fig. 3 and Fig. 4 depict the concentration dependences of real and imaginary parts of the refractive index for a wavelength of $633 \mathrm{~nm}$.

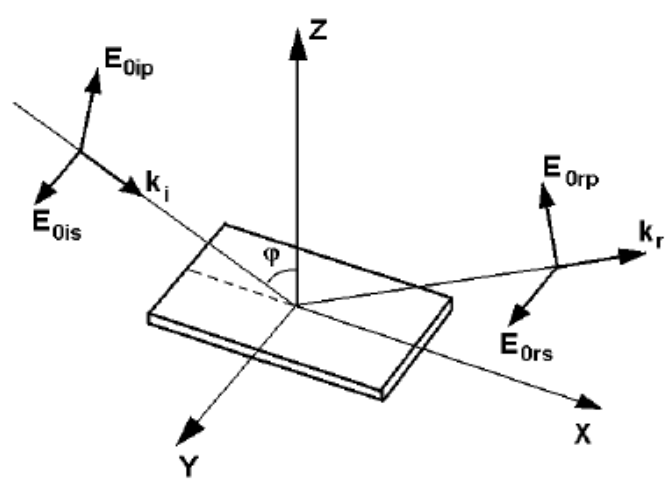

Fig. 1. Scheme of ellipsometric experiments.

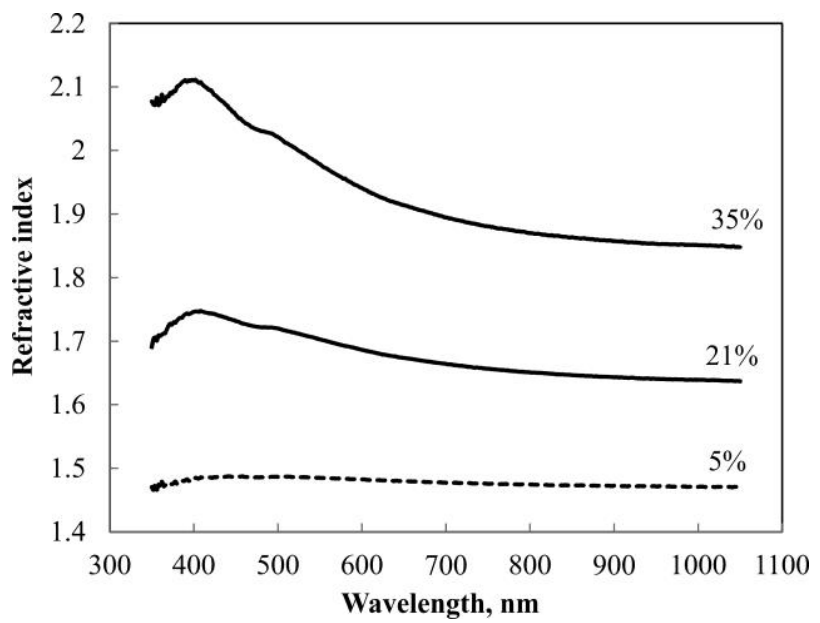

Fig. 2. Spectra of the real part of a complex refractive index for magnetic fluids various concentrations.

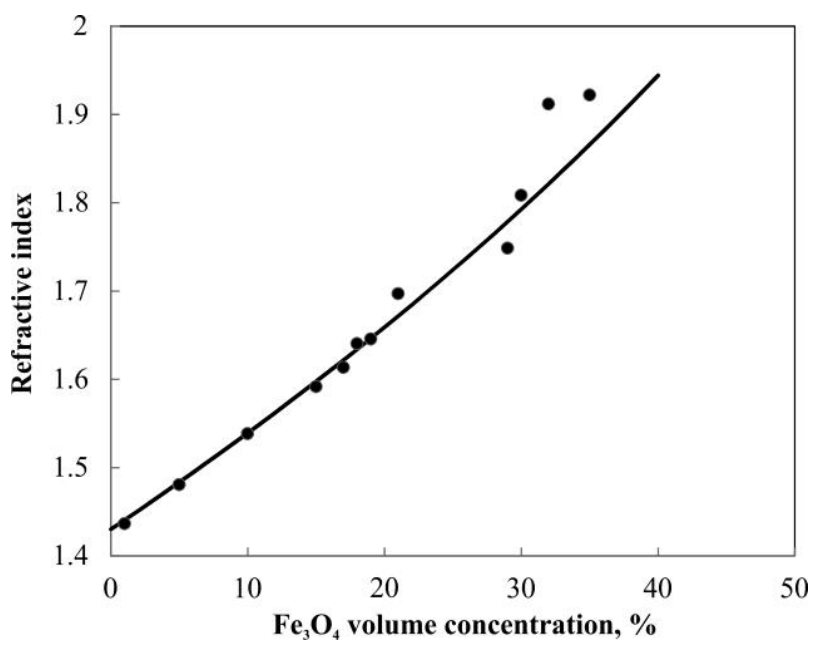

Fig. 3. Dependence of real part of refractive index on the concentration of the magnetite (dots - experimental data; line Maxwell Garnett effective medium approximation).

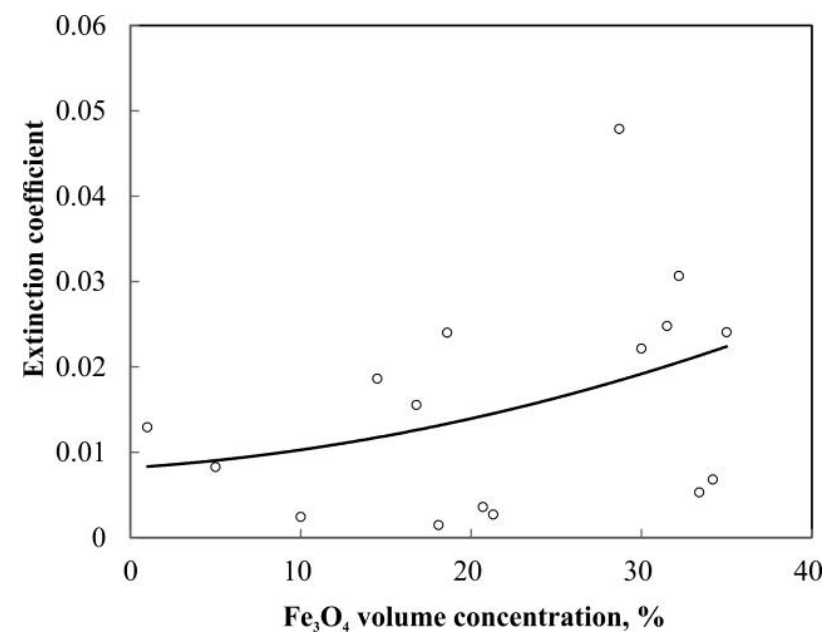

Fig. 4. Dependence of imaginary part of refractive index on the concentration of the magnetite.

\section{Results and Discussion}

For a theoretical estimate of the concentration dependence of magnetic fluid refractive index various approximations of the effective medium theory were used. One of the most famous and widely used models of an effective medium are the following models: Maxwell (known from Landau's book [11]), as well as the Maxwell-Garnett and Bruggeman's models [12]. To determine the effective dielectric constant at high particle concentrations, the Maxwell-Garnett (WienerWagner) equation is used, which is valid for volume concentration $f>1 / 3$ :

$$
\frac{\varepsilon_{e f f}-\varepsilon_{0}}{\varepsilon_{e f f}+2 \varepsilon_{0}}=f \frac{\varepsilon_{1}-\varepsilon_{0}}{\varepsilon_{1}+2 \varepsilon_{0}}
$$

Or Bruggeman's equation:

$$
(1-f) \frac{\varepsilon_{0}-\varepsilon_{e f f}}{\varepsilon_{0}+2 \varepsilon_{e f f}}+f \frac{\varepsilon_{1}-\varepsilon_{e f f}}{\varepsilon_{1}+2 \varepsilon_{e f f}}=0 .
$$


Where $\varepsilon_{0}$ and $\varepsilon_{1}$ are dielectric constants of liquid medium and particle material (magnetite) respectively. The Maxwell-Garnett and Bruggeman's equations give the same results for $f=0 \quad\left(\varepsilon_{\text {eff }}=\varepsilon_{0}\right)$ and for $f=1$ ( $\varepsilon_{\text {eff }}=\varepsilon_{1}$ ), but for intermediate values of $f$ the curves are different. The relationship between the effective dielectric permittivity $\widetilde{\varepsilon}=\varepsilon^{\prime}+i \varepsilon^{\prime \prime}$ and the refractive index $\tilde{n}=n+i k$ of the system is given by the equations:

$$
n=\sqrt{\frac{\varepsilon^{\prime}+\sqrt{\varepsilon^{\prime 2}+\varepsilon^{\prime 2}}}{2}}, \quad k=\frac{\varepsilon^{\prime \prime}}{2 n} .
$$

We calculated the concentration dependence of the refractive index for the above-listed models and the values of the real and imaginary parts of the refractive index of magnetite particles, with the best coincidence between the experimental data and calculations. In Fig. 3 the results of calculations of the real part of the refractive index are shown. It is obvious that in the field of low concentrations $(f<10 \%)$ all the approximations give approximately the same results. It should be noted that the value of the refractive index at which the experimental and calculated data are equal to $\tilde{n}=2.9+0.015 i$ is significantly different from the tabulated data for bulk magnetite [13] at the same wavelength $(633 \mathrm{~nm})$.

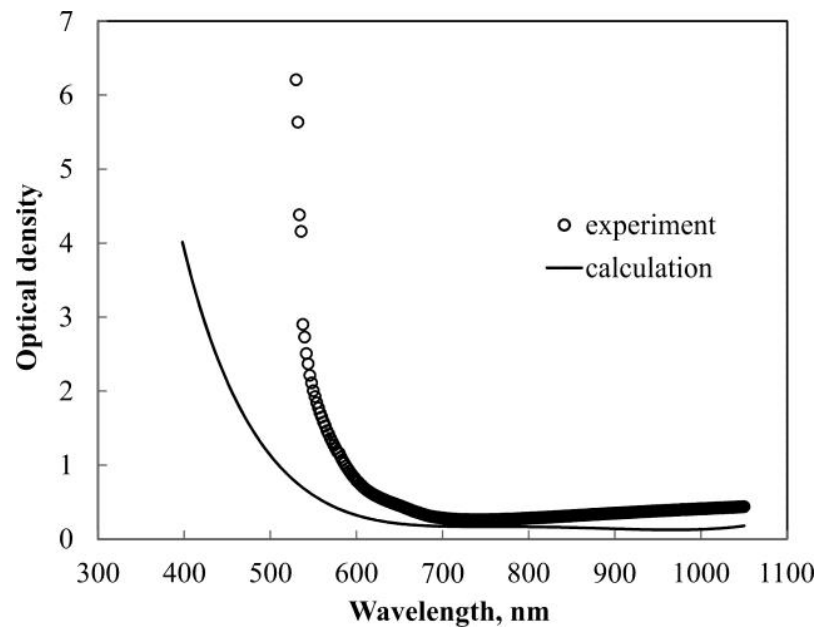

Fig. 5. Spectra of optical density of magnetic fluid (volume concentration of magnetite $5 \%$, sample thickness is $0.04 \mathrm{~mm}$ ).

Optical density spectra were calculated using the obtained spectral dependences of real and imaginary parts of the refractive index of a highly concentrated magnetic fluid. It is well known that [14], the extinction of light passing through the dispersion, as subject to single scattering of light (i.e., not a very large concentration of particles) is described by Bouguer-Lambert law:

$$
I=I_{0} \exp (-\sigma N l),
$$

where $\sigma$ is cross-section of light extinction, $l$ is the radiation path length in a cell, $N$ is numeric concentration of particles, and $I_{0}$ is the intensity of the incident light. By definition, the optical density of the medium is:

$$
D=\log \frac{I_{0}}{I}=0.43 \sigma N l .
$$

Let us to consider the contribution of light extinction and scattering by magnetic colloids in the spectral dependence of the optical properties. The cross section of the light extinction is made up of the scattering efficiency factor $Q_{s c a}$ and absorption efficiency factor $Q_{a b s}$ :

$$
\sigma=\pi a^{2}\left(Q_{s c a}+Q_{a b s}\right)
$$

For small Rayleigh's particles expressions for the efficiency factors are in form [14]:

$$
\begin{gathered}
Q_{s c a}=\frac{8}{3}\left(\frac{2 \pi a}{\lambda}\right)^{4}\left|\frac{m^{2}-1}{m^{2}+2}\right|^{2} . \\
Q_{a b s}=\frac{8 \pi a}{\lambda} \operatorname{Im}\left(\frac{m^{2}-1}{m^{2}+2}\right)^{2} .
\end{gathered}
$$

Where $a, m-$ are the size of particles and relative refractive index respectively. Measurements of the spectral dependencies of the optical density were performed using spectral ellipsometric apparatus "Ellipse 1891" in the mode of the spectral photometer. The weakening of the light transmitted through the sample was detected by a photodetector, and received data is automatically calculated dependence of $D(\lambda)$. Fig. 5 shows the calculation of the optical density spectrum of a magnetic fluid with a concentration of $5 \%$ by formulas (710) and the results of experimental measurement of this spectrum. The difference between experimental and calculated curves can be explained by the polydispersity of the sample.

\section{Conclusions}

Spectral dependences of real and imaginary parts of the refractive index of a magnetic fluid based on kerosene with magnetite nanoparticles are obtained. It is shown that the concentration dependence of the real part of the refractive index is properly described by the Bruggeman's equation in the effective medium theory. Extrapolation of the values $\operatorname{Re}(\tilde{n})$ for bulk magnetite gives a higher value than known tabulated data. Calculation of the spectral dependence of the extinction of thin films of a concentrated magnetic fluid shows that extinction is mainly determined by light absorption.

This work was supported by Russian Foundation for Basic Researches (project No.16-03-00054).

\section{References}

1. S.Y. Yang, J.J. Chieh, H.E. Horng Appl. Phys. Lett. 84, 5204 (2004)

2. U. Buchenau, I. Muller, Solid State Comm. 11, 1291 (1972)

3. S.Y. Yang, Y.F. Chen, H.E. Horng, Chin-Yih Hong, W.S. Tse et al. Appl. Phys. Lett. 81, 4931 (2002)

4. E.Ya. Levitin, N.G. Kokodiy, V.A. Timanjuk, I.O. Vedernikova, T.M. Chan Inorganic Mater. 50, 8 (2014) 
5. H. E. Horng, Chin-Yih Hong, S.Y. Yang, H.C. Yang Appl. Phys. Lett. 82, 2434 (2003)

6. Xiaopeng Fang, Yimin Xuan, Qiang Li Nanoscale Res. Lett. 6, 237 (2011)

7. Yu.I. Dikansky, A.R. Zakinyan, A.N. Tyatyushkin Phys. Rev. E 84, 031402 (2011)

8. K.T. Wu, Y.D. Yao, H.K. Huang J. Magn. Magn. Mater. 209, 246 (2000)

9. K.V. Erin, S.A. Kunikin Optics and Spectroscopy 104, 277 (2011)

10. H. Fujiwara Spectroscopic Ellipsometry: Principles and Applications (John Wiley \& Sons, 2007)

11. L.D. Landau and E.M. Lifshitz Electrodynamics of Continuous Media (Pergamon Press, Oxford, 1960)

12. T.C. Choy Effective Medium Theory (Oxford: Clarendon Press, 1999)

13. M.R. Querry Optical Constants (Aberdeen, 1985)

14. C.F. Bohren, D. R. Huffman Absorption and Scattering of Light by Small Particles (Wiley, 2007) 